\title{
Homocysteine Levels and Cardiovascular Risk: Potential Predictor of Morbidity and Mortality
}

\author{
Ivan Lozada Martinez ${ }^{1 *}$, Daniela Torres Llinás ${ }^{2}$ and Andres Llamas Nieves ${ }^{2}$ \\ ${ }^{1}$ Director of Medical-Surgical Research Center, School of Medicine, University of Cartagena, Cartagena Colombia \\ ${ }^{2}$ Researcher, Medical-Surgical Research Center, School of Medicine, University of Cartagena, Cartagena, Colombia
}

*Corresponding author: Ivan David Lozada Martinez, Director of Medical-Surgical Research Center, School of Medicine, University of Cartagena, Cartagena, Colombia

Homocysteine is a sulfurated amino acid, deriving out of methionine from the metabolism of the methyl group [1]. It is a non-protein substance that has been associated with the dynamics of endothelial function, platelet activation, and leukocyte interaction, with the capacity to intensify the inflammatory response in the cardiovascular system, it is therefore considered a potential cardiovascular risk marker [2]. Some studies have evaluated homocysteine in risk groups, where it has been found that the elevation of its levels (hyperhomocysteinemia) is correlated as a predictor of coronary artery disease, peripheral arterial disease, ictus, deep vein thrombosis, and elevated blood pressure during pregnancy [3-5]. Taking into account that cardiovascular diseases remain a major cause of morbidity, mortality, and disability, with high economic costs around the world [6], research on biomarkers that can predict vascular injury and serve as therapeutic targets is extremely necessary.

Zheng, et al. [7] conducted a study analyzing homocysteine levels for one year in 3229 patients who had suffered a stroke. They found that hyperhomocysteinemia was an independent predictor of mortality (HR: 1.70; $95 \% \mathrm{Cl}: 1.01-2.88, \mathrm{p}<0.05)$, also they predicted the risk of recurrence of stroke, mainly in those individuals with low platelet levels [7]. These results are consistent with those of the study conducted by Khan, et al. [8], where through the use of high-resolution metabolomic studies, they concluded that in patients with high cardiovascular risk, there is hyperactivity in the metabolic pathways of homocysteine and cysteic acid, prom- ising then to be a non-invasive marker of morbidity with a strong cause-effect relationship [8].

Based on the results of this type of studies, studies aimed at counteracting hyperhomocysteinemia with vitamin B6, vitamin B12 and folate have been postulated, to assess the impact of this intervention on the process of atherosclerosis and decreased plasma homocysteine levels, finding favorable results in murine knockout models [9], but unfortunately not in humans [10]. However, very few clinical trials have evaluated this intervention [10], in addition to being obsolete, so it cannot be argued that it is not effective.

Toya, et al. [5] conducted a study in which they evaluated the association between plasma homocysteine levels with peripheral microvascular endothelial dysfunction, and their impact on major adverse cardiovascular events, through peripheral arterial tonometry of reactive hyperemia [5]. They found that those patients who had hyperhomocysteinemia, mostly were older, male, and with a higher number of comorbidities, compared to those who had low homocysteine levels. Hyperhomocysteinemia was strongly associated with an increased risk of major adverse cardiovascular events (HR: 3.65; $95 \% \mathrm{Cl}: 1.41-9.48, \mathrm{p}=0.01)$, even after adjusting the values by age [5].

There is still much to investigate about hyperhomocysteinemia, there is published evidence where it has been found that this condition is linked to insulin resistance [11], atrial fibrillation [12], disorders in sexual activity [13], and cardiometabolic health deficit in young

Citation: Martinez IL, Llinás DT, Nieves AL (2021) Homocysteine Levels and Cardiovascular Risk: Potential Predictor of Morbidity and Mortality. Int Arch Cardiovasc Dis 5:035. doi.org/10.23937/26433966/1710035

Accepted: February 04, 2021: Published: February 06, 2021

Copyright: (c) 2021 Martinez IL, et al. This is an open-access article distributed under the terms of the Creative Commons Attribution License, which permits unrestricted use, distribution, and reproduction in any medium, provided the original author and source are credited. 
people [14], so its role between the interaction of the cardiovascular system and other systems is not entirely elucidated, however, to find an effective and cost/benefit-effective technique, would be an innovative achievement with a high impact on the problem of the burden of the disease currently faced by many researchers and scientific societies. Likewise, it should be sought to improve which substances would manage to regulate homocysteine levels, and therefore, reduce the risk of suffering fatal cardiovascular events.

\section{Financial Support}

None.

\section{Conflict of Interest}

None.

\section{Authors Contribution}

All authors have contributed for this manuscript.

\section{References}

1. Wei M, Wang L, Liu YS, Zheng MQ, Ma FF, et al. (2019) Homocysteine as a potential predictive factor for high major adverse cardiovascular events risk in female patients with premature acute coronary syndrome. Medicine (Baltimore) 98: e18019.

2. Oudi ME, Aouni Z, Mazigh C, Khochkar R, Gazoueni E, et al. (2010) Homocysteine and markers of inflammation in acute coronary syndrome. Exp Clin Cardiol 15: e25-e28.

3. Cacciapuoti F (2011) Hyper-homocysteinemia: A novel risk factor or a powerful marker for cardiovascular diseases? Pathogenetic and therapeutical uncertainties. J Thromb Thrombolysis 32: 82-88.

4. Homocysteine Studies Collaboration (2002) Homocysteine and risk of ischemic heart disease and stroke: A meta-analysis. JAMA 288: 2015-2022.

5. Toya T, Sara JD, Lerman B, Ahmad A, Taher R, et al. (2020) Elevated plasma homocysteine levels are associated with impaired peripheral microvascular vasomotor response. Int J Cardiol Heart Vasc 28: 100515.
6. Mensah GA, Roth GA, Fuster V (2019) The Global Burden of Cardiovascular Diseases and Risk Factors: 2020 and Beyond. J Am Coll Cardiol 74: 2529-2532.

7. Zhenga $X$, Guoa D, Penga $H$, Zhonga $C$, Bua $X$, et al. (2019) Platelet counts affect the prognostic value of homocysteine in acute ischemic stroke patients. Atherosclerosis 285: 163-169.

8. Khan A, Choi Y, Back JH, Lee S, Jee SH, et al. (2020) High-resolution metabolomics study revealing l-homocysteine sulfinic acid, cysteic acid, and carnitine as novel biomarkers for high acute myocardial infarction risk. Metabolism 104: 154051.

9. Hofmann MA, Lalla E, Lu Y, Gleason MR, Wolf BM, et al. (2001) Hyperhomocysteinemia enhances vascular inflammation and accelerates atherosclerosis in a murine model. $\mathrm{J}$ Clin Invest 107: 675-683.

10. Woo KS, Chook P, Lolin YI, Sanderson JE, Metreweli C, et al. (1999) Folic acid improves arterial endothelial function in adults with hyperhomocystinemia. J Am Coll Cardiol 34: 2002-2006.

11. Ebrahimpour A, Vaghari-Tabari M, Qujeq D, Moein S, Moazezi Z (2018) Direct correlation between serum homocysteine level and insulin resistance index in patients with subclinical hypothyroidism: Does subclinical hypothyroidism increase the risk of diabetes and cardio vascular disease together? Diabetes Metab Syndr 12: 863-867.

12. Kubota Y, Alonso A, Heckbert SR, Norby FL, Folsom AR (2019) Homocysteine and Incident Atrial Fibrillation: The Atherosclerosis Risk in Communities Study and the Multi-Ethnic Study of Atherosclerosis. Heart Lung Circ 28: 615-622.

13. Yang HF, Kao TW, Lin YY, Shih MT, Wu LW, et al. (2017) Does Serum Homocysteine Explain the Connection Between Sexual Frequency and Cardiovascular Risk? J Sex Med 14: 910-917.

14. Rudreshkumar KJ, Majumdar V, Nagaraja D, Christopher $R$ (2018) Relevance of plasma levels of free homocysteine and methionine as risk predictors for ischemic stroke in the young. Clin Nutr 37: 1715-1721. 